\title{
“CRISPR Edited Haematopoietic Stem Cells”: Solution to Sickle Cell Disease - A Systematic Review
}

\author{
Prerana $\mathbf{G}^{1}$, Raajasiri Iyengar ${ }^{2}$ and Gaurav ${ }^{3 *}$ \\ ${ }^{1}$ House Surgeon, NSVK Sri Venkateshwara Dental College and Hospital, Bangalore, \\ Karnataka, India \\ ${ }^{2}$ Final Year Undergraduate Student, NSVK Sri Venkateshwara Dental College and \\ Hospital, Bangalore, Karnataka, India \\ ${ }^{3}$ Consultant Oral Physician and Maxillofacial Radiologist, Assistant Professor, \\ Department of Oral Medicine and Maxillofacial Radiology, NSVK Sri Venkateshwara \\ Dental College and Hospital, Bangalore, Karnataka, India
}

*Corresponding Author: Gaurav, Consultant Oral Physician and Maxillofacial Radiologist, Assistant Professor, Department of Oral Medicine and Maxillofacial Radiology, NSVK Sri Venkateshwara Dental College and Hospital, Bangalore, Karnataka, India.

\begin{abstract}
Background: Sickle cell disease is caused by mutations in the beta-globin gene, resulting in misshapen red blood cells. Reactivation of fetal hemoglobin ( $\mathrm{HbF}$ ) is being pursued as a treatment strategy for hemoglobinopathies. The approach is based on hematopoietic stem cells that "engraft," or settle, in the bone marrow to produce red blood cells with functional hemoglobin [1].

Aim of the Study: To determine the therapeutic potential of hematopoietic stem and progenitor cells (HSPCs) edited with the CRISPR-Cas9 nuclease and evaluate recapitulation of naturally occurring mutations identified in individuals who express hereditary persistence of $\mathrm{HbF}$

Research Question: Can CRISPER edited HSPCs offer a solution for the treatment of sickle cell disease?

Materials and Methods: With the Medline database taken as a source for authenticated scientific research data, articles were selected having undergone randomized control trial. Out of these, articles (studies) were chosen which met the criterion for systematic review.

Result and Conclusion: Treatment of SCD mostly includes dealing with the complications of sickle cell anemia as they occur. It might include antibiotics, vitamins, blood transfusions, pain-relieving medicines, other medications and possibly surgery, such as to correct vision problems or to remove a damaged spleen. No treatment modality has offered a complete cure of the disease. CRISPR is a highly precise gene-editing tool that relies on guide RNA molecules to direct a scissor-like Cas9 enzyme to just the right spot in the genome to correct the misspelling [2]. The gene-editing treatment involves removing bone marrow from a patient, modifying the HSCs outside the body using CRISPR gene-editing tools, and then returning them back to the patient [3]. CRISPR plays a key role in treating SCD by correcting the mutation in the beta globin gene, promoting the production of fetal hemoglobin and enhancing the recovery rate [4].
\end{abstract}

Keywords: Haematopoietic; Sickle Cell Anemia (SCA); CRISPR

\section{Introduction}

Sickle cell anemia (SCA) as the literature talks is a genetic disorder that results in an abnormality in the hemoglobin predominantly due to the mutation of 'spectrin' protein which leads to a rigid, sickle-like shape of RBCs under certain circumstances. It has established itself as a potentially life threatening condition. Though numerous treatment modalities have been tried so far in order to cure this particular genetic disorder, unfortunately no treatment in specific has been able to turn the tables around [5]. There has been a lot of ambiguity regarding the role of gene editing and its significance in treating SCD [3]. Hence, the present study aims to determine the therapeutic potential of haematopoietic stem and progenitor cells (HSPCs) edited with CRISPR [4] - Cas9 nuclease in treating SCD and the research question - "can CRISPR edited HSPCs offer a solution for the treatment of sickle cell disease?" 


\section{Materials and Methods}

Various researches and studies have documented the CRISPR-Cas9 to be sensitive and specific. With this fact in mind, a literature based systematic review was carried out to fulfill the aim of the study. With Cochrane collaboration taken as source for authenticated scientific research data, about 30 research articles were selected having undergone a randomized control trial. Out of these, the articles were screened and finally 25 articles were chosen which met the criterion for systematic review. The selection criteria have been described below.

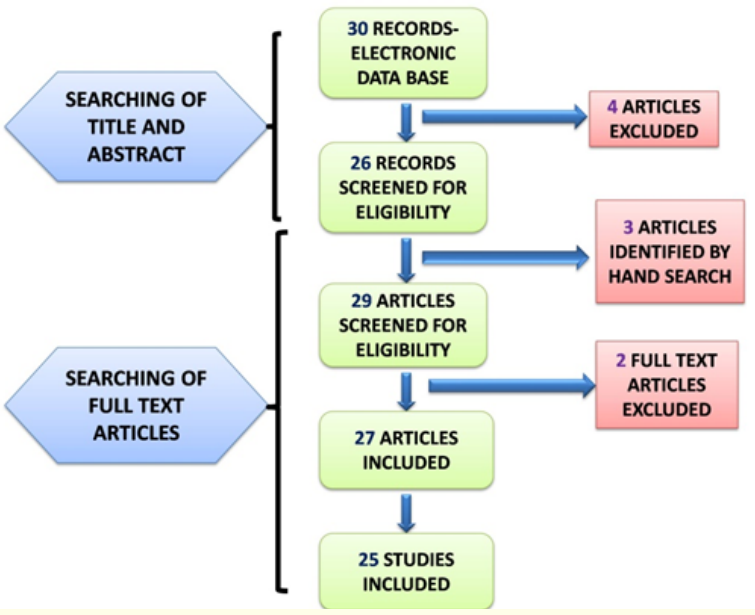

Figure 1

\section{Result}

The actual need for this systematic review was laid on the foundation of following conclusions which were drawn from the final 25 studies which were selected, viz; despite all the years of research there has been no valid treatment over time and SCD can be classified as an incurable disease as it is genetic in its etiopathogenesis. The increase in number of negative clinical trials of pharmacological treatment for SCD highlights the need for new molecular targets. Although in the phase of clinical trials, CRISPRCa9 represents new promising potential targets for the next-future drugs.

The systematic review conducted in order to document the significance of the CRISPR-Cas9 in the treatment of SCD [4] was found to be highly significant although still in clinical trials as the new modality comes up with the following major advantages:

- Correcting the mutation in the beta globin gene.

- Promoting the production of fetal hemoglobin.

- Enhancing the recovery rate.

\section{Discussion}

Sickle cell disease is an autosomal recessive disorder. Sickle cell anemia is the most severe form of sickle cell disease and is the homozygous state for hemoglobin S. Sickle cell anemia is prevalent in Africa, the Middle East, and parts of India. In sickle cell disease, hemoglobin precipitates as insoluble crystals which lead to an abnormal shape and size of RBCs with subsequent phagocytosis of the effected corpuscles. A point mutation in the beta globin chain of the hemoglobin causes sickle cell disease. Specifically, it occurs when a single base from $\mathrm{A}$ to $\mathrm{T}$ in the codon for glutamic acid at position 6 is changed to valine of the beta globin. If this mutation affects both of the beta globin chains, sickle cell anemia occurs; if only one chain is affected, it results in the sickle cell trait. The vasoocclusive crises are triggered by: Hypoxia, Cold weather, Infections, Acidosis, Dehydration, Alcohol, Stress and Pregnancy.

The mutation's presence in both chains causes a change in hemoglobin. Normal hemoglobin is soluble, and it does not precipitate in the presence of hypoxia, low $\mathrm{pH}$, and dehydration. The polymerization of the sickle cell hemoglobin molecules inside the RBCs is responsible for the sickling. This polymerization with subsequent aggregation takes place when the sickle cell hemoglobin is deoxygenated. Initial aggregation and polymerization is a reversible process. It occurs while the hemoglobin is deoxygenated and reverses with oxygenation. The sickle or holly leaf shape of the RBCs conforms to the shape of the polymerized hemoglobin. However, multiple cycles of sickling cause damage to the RBCs' cell membranes, making them prone to phagocytosis by the macrophages.

This phagocytosis leads to the destruction and reduction of RBC count and hence results in anemia. Reversibly sickled cells revert to normal shape with oxygenation and continue to perform their functions, but when the hemoglobin is irreversibly polymerized oxygenation cannot reverse the pathology. The deformed sickle cells have a strong adherence to the endothelium, leading to vasoocclusive crisis.

\section{What is CRISPR-cas9?}

CRISPR is an acronym that stands for Clustered Regularly Interspaced Short Palindromic Repeats. Cas9 or CRIPSR associated protein 9 is an enzyme that uses CRISPR sequences as a guide to recognize and cleave specific strands of DNA that are complementary to the CRISPR sequence. The technology creates a small piece of RNA with a short "guide" sequence that attaches (binds) to a specific target sequence of DNA in a genome. The RNA also binds to the Cas9 enzyme. As in bacteria, the modified RNA 


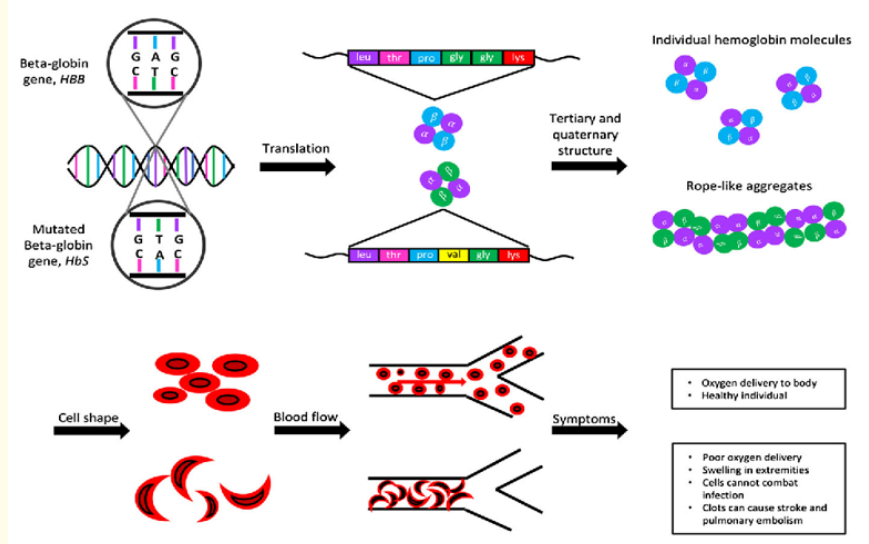

Figure 2

is used to recognize the DNA sequence, and the Cas9 enzyme cuts the DNA at the targeted location. Although Cas9 is the enzyme that is used most often, other enzymes (for example Cpf1) can also be used. Once the DNA is cut, researchers use the cell's own DNA repair machinery to add or delete pieces of genetic material, or to make changes to the DNA by replacing an existing segment with a customized DNA sequence [6].

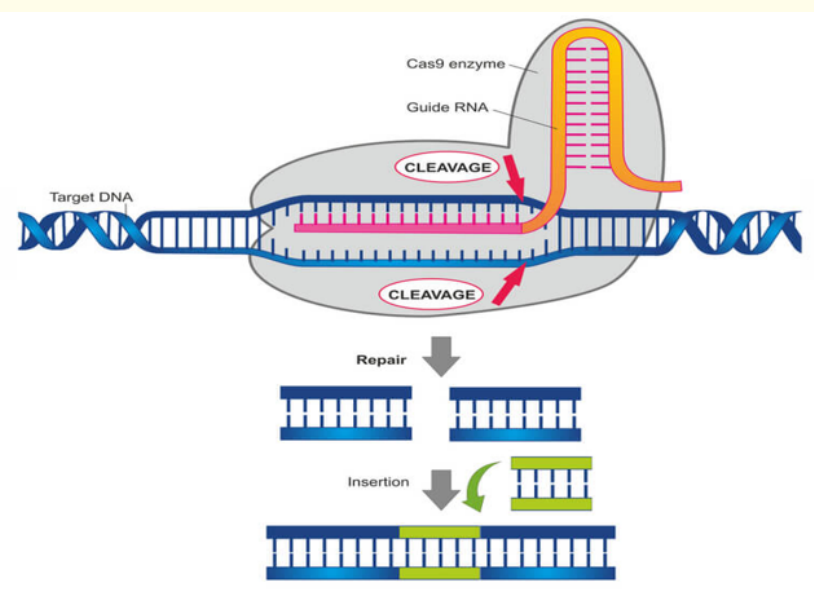

Figure 3

Mechanism of action of crispr-cas9 in treating sickle cell disease

Since sickle cell disease is a genetic disorder, there are two mechanisms through which CRISPR works:

1. Using a guide RNA, the Cas9 enzyme can target and repair the faulty beta globin gene. At the point of mutation, thymine is replaced with adenine and the base is corrected.
The corrected gene is now replaced and normal blood cells are produced.

2. Cas9 enzyme also promotes the production of fetal hemoglobin by breaking a gene that encodes for a repressor such as BAC11A such that the production of fetal hemoglobin is no longer blocked and sickling of RBCs are prevented.



Figure 4

Before the doctors can inject the edited cells back into the patient's bone marrow they have to damage their other stem cells using radiation and chemotherapy. If they don't, the unedited stem cells will continue to produce sickled red blood cells faster than the edited cells can produce healthy ones. To give the fetal haemoglobin an advantage and make sure healthy, round blood cells get ahead, they injure the original stem cells that produce sickle cells.

After that, it becomes a waiting game. They wait for fetal haemoglobin to increase and for the amount of sickled blood cells to decrease. For the treatment to be worth it for the patient, it has to significantly improve the patient's quality of life. There must be a functional payout, a long term alleviation of the condition.

\section{Conclusion}

Treatment of SCD mostly includes dealing with the complications of sickle cell anemia as they occur. It might include antibiotics, vitamins, blood transfusions, pain-relieving medicines, other medications and possibly surgery, such as to correct vision problems or to remove a damaged spleen. No treatment modality has offered a complete cure of the disease. CRISPR is a highly precise gene-editing tool that relies on guide RNA molecules to direct a scissor-like Cas 9 enzyme to just the right spot in the genome to correct the misspelling. The gene-editing treatment 
involves removing bone marrow from a patient, modifying the HSCs outside the body using CRISPR gene-editing tools, and then returning them back to the patient. CRISPR can play a key role in treating SCD by correcting the mutation in the beta globin gene, promoting the production of fetal hemoglobin and enhancing the recovery rate. The use of CRISPR can be extended to encompass the definitive therapies for other inherited haemoglobinopathies, which has until now remained largely unsolved, opening doors for novel therapeutics using CRISPR.

When used ethically, CRISPR-Cas9 has tremendous potential to revolutionize the treatment of many genetic disorders While significant progress has been made, there is still a great deal of ground to uncover with CRISPR-Cas9 technology.

\section{Bibliography}

1. Wu X., et al. "Genome-wide binding of the CRISPR endonuclease Cas9 in mammalian cells". Nature Biotechnology 32.7 (2014): 670-676.

2. Doudna JA and Charpentier E. "Genome editing. The new frontier of genome engineering with CRISPR-Cas9". Science 346.6213 (2014): 1258096.

3. Li Y., et al. "A versatile reporter system for CRISPR-mediated chromosomal rearrangements". Genome Biology 16 (2015): 111.

4. Wu Y., et al. "Highly efficient therapeutic gene editing of human hematopoietic stem cells". Nature Medicine (2019).

5. Yin H., et al. "Genome editing with Cas9 in adult mice corrects a disease mutation and phenotype". Nature Biotechnology 32.6 (2014): 551-553.

6. Renaud JB., et al. "Improved genome editing efficiency and flexibility using modified oligonucleotides with TALEN and CRISPR-Cas9 nucleases". Cell Reports 14.9 (2016): 2263-2272.

\section{Assets from publication with us}

- Prompt Acknowledgement after receiving the article

- Thorough Double blinded peer review

- Rapid Publication

- Issue of Publication Certificate

- High visibility of your Published work

Website: www.actascientific.com/

Submit Article: www.actascientific.com/submission.php

Email us: editor@actascientific.com

Contact us: +919182824667

Citation: Gaurav., et al. “"CRISPR Edited Haematopoietic Stem Cells”: Solution to Sickle Cell Disease - A Systematic Review”. Acta Scientific Dental Sciences 4.4 (2020): 103-106. 